\title{
TCL1 promotes blastomere proliferation through nuclear transfer, but not direct phosphorylation, of AKT/PKB in early mouse embryos
}

\author{
Cell Death and Differentiation (2008) 15, 420-422; doi:10.1038/sj.cdd.4402228; published online 28 September 2007
}

Dear Editor,

The T-cell leukemia/lymphoma 1, TCL1, a gene physiologically expressed during T-cell and B-cell development and involved in the pathogenesis of T-cell and B-cell leukemias/ lymphomas, ${ }^{1}$ has recently gained novel interest by the discovery that it plays a key regulatory role in the maintenance of the proliferation versus differentiation balance of embryonic stem cells (ES). In ES, in fact, TCL1 is directly activated by $O c t 3 / 4^{2}$ and is among the very few genes that are required for the mitotic self-renewal state, and the downregulation of which triggers ES differentiation. ${ }^{3}$ The expression of TCL1 was also characterized during preimplantation embryo development, namely in the cells from which ES are derived, where it was found to shuttle between blastomere cortical regions and the nucleus by a cell cycle-dependent fashion and to be required for early blastomere proliferation, but not the acquisition of first embryonal differentiation traits. ${ }^{4}$ TCL1 promotes cell proliferation by heterodimerization with AKT/ $\mathrm{PKB}$, a serine/threonine kinase having a central role in the signaling pathways controlling cell proliferation and survival, and mediates both the transphosphorylation of AKT at the Ser472/473 residue ${ }^{5}$ and the transfer of phosphorylated AKT to the nucleus. ${ }^{6}$ However, it is still unclear if these TCL1 functions are both required for the TCL1-mediated promotion of normal/neoplastic cell growth. We have now addressed this question by studying the preimplantation mouse embryo, a physiological and thoroughly studied system, allowing the intracellular traffic to be analyzed with high spatial and temporal resolution.

We have first determined the state of AKT phosphorylation in early embryos by immunofluorescence analysis, revealing the presence of Ser473- and Thr308-phosphorylated AKT in both one-cell and two-cell embryos (Figure 1a and Supplementary Figure S1). Phosphorylated AKT was never localized in pronuclei at the one-cell stage and acquired a prominent nuclear localization in mid two-cell embryos, suggesting that this is the stage at which the AKT-mediated function of TCL1 is first initiated. Therefore, the TCL1 localization in mid onecell embryo pronuclei ${ }^{4}$ is independent of AKT. Further study is required to characterize this feature. To study the regulation of AKT phosphorylation during early embryo development, we have probed the functional relevance of kinases known to regulate AKT phosphorylation, including phosphatidylinositol3-kinase (PI3K) and phosphoinositide-dependent kinase 1 (PDK1), ${ }^{7}$ by allowing one-cell embryos to develop in vitro in the continuous presence of kinase inhibitors. Embryo exposure to either $10-20 \mu \mathrm{M}$ LY294002 or 6-24 nM Wortmannin negatively affected preimplantation development from the four-cell stage (Figure 1b), in agreement with previous observations. ${ }^{8}$ Interestingly, both inhibitors had no apparent effect on the occurrence of the first embryo cleavage, even when the inhibitor concentration in the medium was raised to extremely high values as $100 \mu \mathrm{M}$ LY294002 or $100 \mathrm{nM}$ Wortmannin (not shown). Embryos exposed to such high inhibitor concentration displayed an altered overall morphology and did not progress beyond the four-cell stage, indicating that the inhibitor had effectively damaged cellular functions (Supplementary Figure S2a). However, neither LY294002 nor Wortmannin had any effect upon the pattern of Ser473/Thr308 AKT phosphorylation and intracellular distribution at the two-cell stage at all concentrations tested (Figure 1c and Supplementary Figure S2a), ruling out the possibility that AKT phosphorylation depended on $\mathrm{PI} 3 \mathrm{~K}$ and thus on de novo synthesis of $\mathrm{PIP}_{3}$ at the beginning of embryo development. We then investigated the role of PDK1 on embryonic AKT phosphorylation. In fact, AKT is phosphorylated on Ser473 by the rictor-mTOR complex, whereas PDK1 phosphorylates AKT on Thr308. ${ }^{9}$ The PDK1 issue was addressed by culturing embryos in the presence of $10-20 \mu \mathrm{M}$ of LY333531, a PKC $\beta$ inhibitor that also inhibits PDK1 in the lower micromolar range. ${ }^{10}$ In spite of its negative effect on later preimplantation development, LY333531 did not apparently affect the first embryo cleavage (Figure 1b) and the AKT phosphorylation pattern of two-cell embryos (Supplementary Figure S2b). Similar results were also obtained with two-cell embryos that had received an injection of anti-PDK1 antibodies at the one-cell stage (Supplementary Figure S2c). We therefore concluded that the phosphorylation state and the intracellular distribution of AKT in two-cell embryos are independent of both PI3K and PDK1 activities, suggesting that AKT is neither de novo Ser473/Thr308-phosphorylated nor dephosphorylated during early stages of preimplantation development. This possibility was tested by determining the total content of phosphorylated AKT in unfertilized eggs and one-cell/two-cell embryos by quantitative immunofluorescence analysis (Figure 1d). The level of Ser473/Thr308phosphorylated AKT appeared not to vary significantly across fertilization and the first and second-cell cycles of preimplantation development. In addition, no difference was found between the phosphorylated AKT content of $\mathrm{TCl}^{-1-}$ and wildtype two-cell embryos, indicating that TCL1 is not required for 


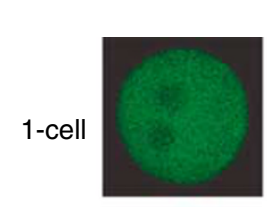

2-cell

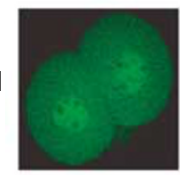

P-Ser473
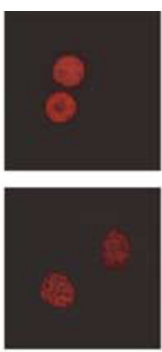
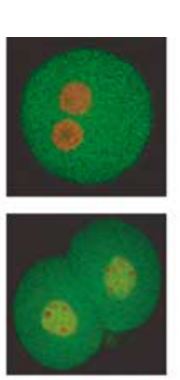

LY 294002 $(10 \mu \mathrm{M})$
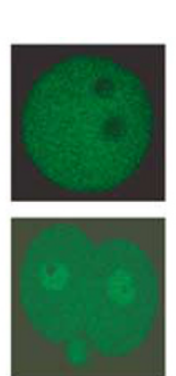

Wortmannin (12 nM)
P-Thr308
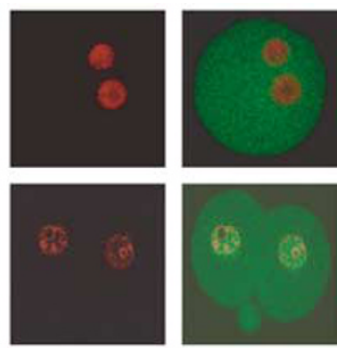

LY333531

$(20 \mu \mathrm{M})$
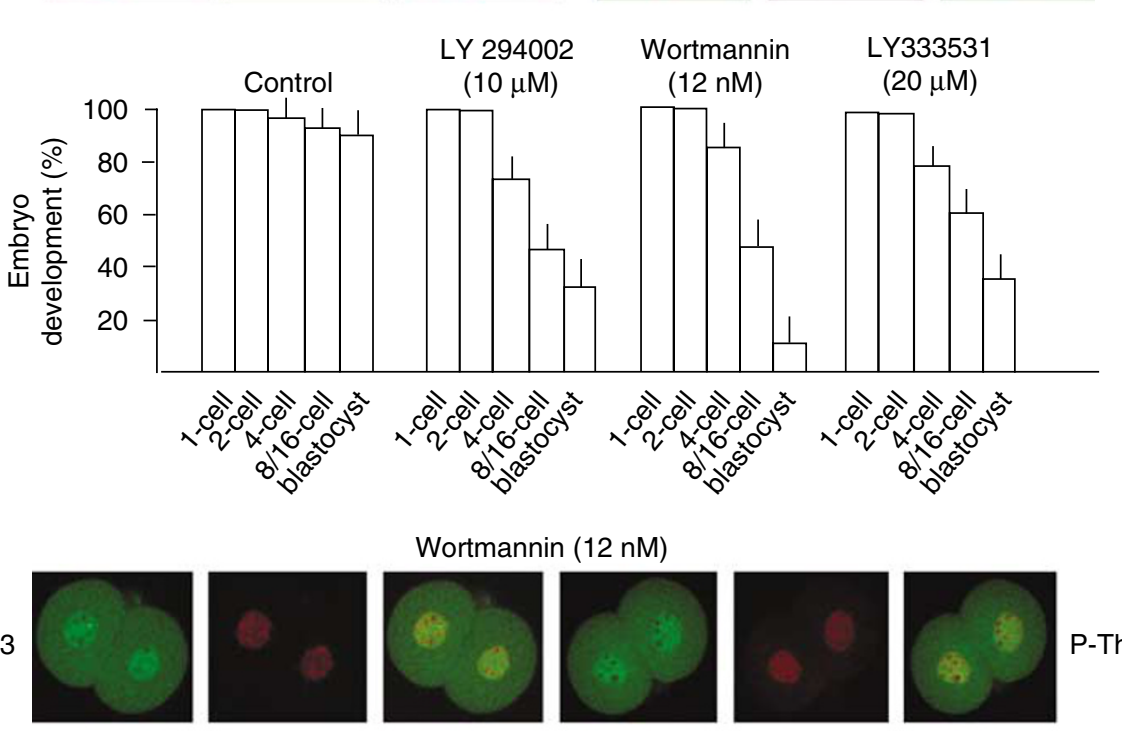

c

$$
\text { Wortmannin (12 nM) }
$$
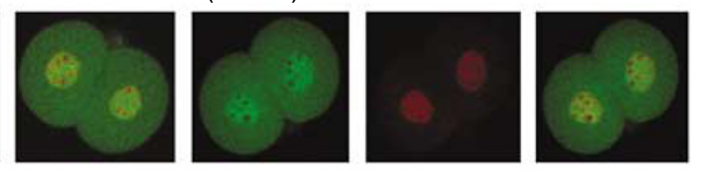

P-Thr308

d

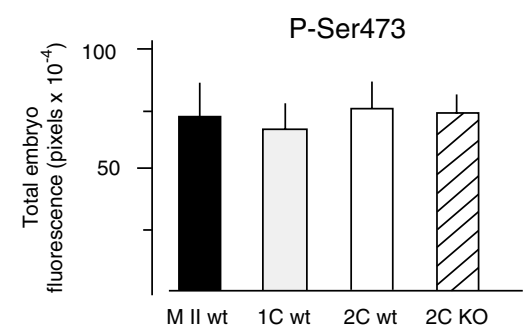

P-Thr308

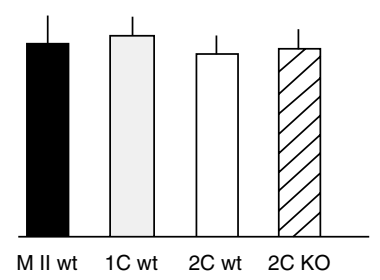

e

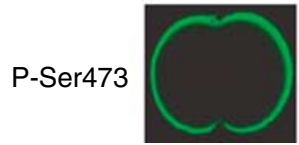

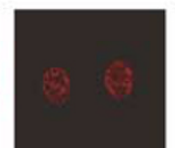

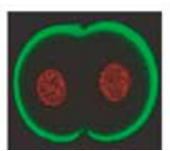

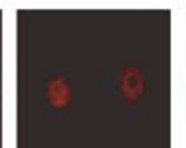

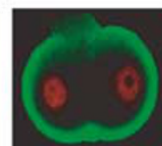

P-Thr308

Figure 1 Regulation of Ser-473/Thr308 AKT phosphorylation during preimplantation development. (a) The intracellular localization of phosphorylated AKT was analyzed by confocal microscopy in wild-type one-cell and two-cell mouse embryos using rabbit monoclonal anti-phospho-AKT(Ser473) and anti-phospho-AKT(Thr308) antibodies (Cell Signalling Technology, Beverly, MA, USA) and an FITC-labeled secondary antibody. Nuclei were stained with propidium iodide (PI). Note the lack of phosphorylated AKT in one-cell embryo pronuclei and the presence in nuclei of two-cell embryos. From left to right: FITC, PI, merge. The same pattern was consistently observed in all embryos analyzed; representative embryos are shown in the panel. (b) To determine the effect of kinase inhibitors on preimplantation embryo development, wild-type one-cell embryos were cultured in vitro in the continuous presence of a PI3K inhibitor (LY294002 or Wortmannin; Cell Signalling Technology) or a PDK1inhibitor (LY333531; AG Scientific, San Diego, CA, USA), and scored daily for their developmental progression and morphology. Bars (mean \pm S.E.M. of three independent experiments having at least 15-20 embryos in each experimental group) indicate the percentage of total embryos that reached the developmental stage indicated in the abscissa. (c) The effect of Wortmannin on the intracellular distribution of Ser473- and Thr308-phosphorylated AKT was determined by allowing one-cell embryos to develop in vitro to the two-cell stage in the continuous presence of $12 \mathrm{nM}$ Wortmannin. Embryos were then immunostained with anti-phospho-AKT(Ser473) or anti-phospho-AKT(Thr308) antibodies and eventually analyzed by confocal microscopy. Note that the nuclear localization of phosphorylated AKT was similar to that of untreated embryos (see a). Features of embryos shown in the panel are representative of three independent experiments, each having at least 15-treated and 15untreated embryos. (d) The total Ser473- and Thr308-phosphorylated AKT content of wild-type (wt) unfertilized eggs (M II, full bars) and embryos at the one-cell (1C, gray bars) and two-cell (2C, empty bars) stages was determined by immunostaining with anti-phospho-AKT (Ser473) or anti-phospho-AKT (Thr308) antibodies, followed by quantitative immunofluorescence analysis (see Supplementary Legends for details), and compared to that of two-cell $T c / 1^{-1-}$ embryos (2C KO, striped bars). (e) The intracellular distribution of Ser473- and Thr308-phosphorylated AKT in two-cell embryos genetically deficient in TCL1 was analyzed by confocal microscopy following immunostaining with anti-phospho-AKT(Ser473) or anti-phospho-AKT(Thr308) antibodies. Features of embryos shown in the panel are representative of a total of 10 $\mathrm{TCl}^{-1-}$ embryos obtained from different breeding pairs 
sustaining AKT phosphorylation in early mouse embryos. Taken together, data obtained with kinase inhibitors and quantitative immunofluorescence analyses support strongly the idea that early mouse embryos inherit fully Ser472/ Thr308-phosphorylated AKT from oogenesis and that initial embryo cleavages are independent on growth factors, making PI3K and PDK1 activities dispensable. This conclusion is in agreement with previous observations that mouse preimplantation embryo development requires PI3K activity from the 8/16-cell stage. ${ }^{8}$

If TCL1 is not relevant to AKT phosphorylation in one-cell and two-cell embryos, is this factor needed for the phosphorylated AKT transfer to nucleus? We have addressed this issue by determining the intracellular distribution of Ser473/ Thr308-phosphorylated AKT in two-cell embryos that were genetically deficient in TCL1. Phosphorylated AKT had a striking cortical localization and was lacking in blastomere nuclei (Figure 1e), pinpointing the cell membrane as an obligatory step among the intracellular movements of phosphorylated AKT. In fact, it is commonly accepted that TCL1 oligomerizes with AKT at the level of cell membrane and that the TCL1-AKT complex is then transferred to nucleus. ${ }^{11}$

In conclusion, early mouse embryos display a physiological dissociation between the TCL1 functions of AKT phosphorylation and phosphorylated AKT transfer to nucleus, pinpointing the latter function as the essential one for the AKT-mediated promotion of cell proliferation. In addition, the present finding that TCL1 enters one-cell embryo pronuclei, while phosphorylated AKT does not, suggests that TCL1 also plays an AKTindependent role(s) at the beginning of embryo development. In light of the recent finding that TCL1 binds the PNPase exoribonuclease, an enzyme that specifically degrades poly$\mathrm{A}^{+} \mathrm{RNAs}^{12}$ it is tempting to hypothesize that this factor is also relevant for the degradation of maternally derived messages.
Acknowledgements. We thank Dr Domenico Grillo for help in quantitative immunofluorescence determinations and $\mathrm{Dr}$ Adriana Bosco for genotyping $\mathrm{TCl}^{-1-}$ mice. This work was supported by grants from Istituto Pasteur - Fondazione Cenci Bolognetti to FM and Associazione Italiana Ricerca sul Cancro and Ministero della Salute to GR.

\section{MT Fiorenza ${ }^{1,2}, S$ Torcia $^{1,2}$, S Canterini $^{1,2}$, A Bevilacqua ${ }^{1,2}$, MG Narducci ${ }^{3}$, G Ragone ${ }^{3}$, $\mathrm{CM} \mathrm{Croce}^{4}, \mathrm{G} \mathrm{Russo}^{3}$ and F Mangia*,1,2}

${ }^{1}$ Istituto Pasteur - Fondazione Cenci Bolognetti, Sapienza University, Rome 00185, Italy;

2 Section of Neuroscience, Department of Psychology, Sapienza University, Rome 00185, Italy;

3 Istituto Dermopatico dell'Immacolata, Istituto di Ricovero e Cura a Carattere Scientifico, Rome 00167, Italy and

4 Human Cancer Genetics Program, Department of Molecular Virology, Immunology, and Medical Genetics, OSU School of Medicine, and Comprehensive Cancer Center, Ohio State University, Columbus, $\mathrm{OH} 43210$, USA

* Corresponding author: Professor F Mangia, Department of Psychology, Section of Neuroscience, Sapienza University of Rome, Via dei Marsi 78, Roma 00185, Italy. Tel.: + 39-0649917784; Fax: + 39-0649917873;

E-mail: franco.mangia@uniroma1.it

\author{
1. Virgilio L et al. Proc Natl Acad Sci USA 1998; 95: 3885-3889. \\ 2. Matoba $\mathrm{R}$ et al. PLOS ONE 2006; 1: e26. \\ 3. Ivanova $\mathrm{N}$ et al. Nature 2006; 442: 533-538. \\ 4. Narducci MG et al. Proc Natl Acad Sci USA 2002; 99: 11712-11717. \\ 5. Kunstle G et al. Mol Cell Biol 2002; 22: 1513-1525. \\ 6. Pekarsky $Y$ et al. Proc Natl Acad Sci USA 2000; 97: 3028-3033. \\ 7. Mora A et al. Semin Cell Dev Biol 2004; 15: 161-170. \\ 8. Lu DP et al. J Cell Sci 2004; 117: 1567-7156. \\ 9. Sarbassov DD et al. Science 2005; 307: 1098-1101. \\ 10. Komander D et al. Structure 2004; 12: 215-226. \\ 11. Teitell MA. Nat Rev Cancer 2005; 5: 640-648. \\ 12. French SW et al. Cancer Lett 2006; 248: 198-210.
}

\title{
Autophagy promotes necrosis in apoptosis-deficient cells in response to ER stress
}

\author{
Cell Death and Differentiation (2008) 15, 422-425; doi:10.1038/sj.cdd.4402234; published online 5 October 2007
}

Dear Editor,

Disruption of endoplasmic reticulum (ER) function is associated with numerous human diseases. ${ }^{1,2}$ It leads to the initiation of a stress response known as the unfolded protein response, whose initial goal is to resolve the ensuing stress; however, when unable to do so, it induces cell death. ${ }^{3,4}$ ER stress-induced cell death has been shown to proceed primarily through apoptosis. It remained unclear whether ER stress is also associated with other forms of cell death. Using bax $^{-/-}$bak $^{-/-}$or $\mathrm{Bcl}-\mathrm{xL}$-overexpressing cells that are defective in apoptosis, here, we show that prolonged ER stress still results in cell death in a fashion resembling necrosis. This necrosis-like cell death is associated with autophagy. In response to ER stress, autophagy is induced in both wild-type and $\mathrm{bax}^{-/-} \mathrm{bak}^{-/-}$cells to a similar extent. Overexpression of wild-type Bax or Bak, as well as ERtargeted Bak does not induce autophagy, indicating that the multi-domain proapoptotic Bcl-2 proteins do not affect autophagy. Inhibition of autophagy results in enhanced cell 